\title{
Zyggie: A Wireless Body Area Network platform for indoor positioning and motion tracking
}

\author{
Antoine Courtay, Mickaël Le Gentil, Olivier Berder, Arnaud Carer, Pascal Scalart, Olivier Sentieys \\ University of Rennes 1 - IRISA \\ Email: firstname.lastname@irisa.fr
}

\begin{abstract}
Nowadays, there is a high demand for human and/or objects monitoring/localizing in the context of applications like Building Information Modeling (BIM), automated drone missions, contextual visits of museum or sports monitoring for instance. While for outdoor positioning accurate and robust solutions (i.e. GPS) exist for many years, indoor positioning is still very challenging. There is also a need of gesture/motion tracking systems that could replace video solutions. We propose in this paper a hardware/software platform named Zyggie that combines both Ultra Wide Band (UWB) technology and Received Signal Strength Indicator (RSSI) for low power accurate indoor positioning and Inertial Measurement Unit (IMU) utilization for motion tracking. Very few industrial/academic existing solutions can simultaneously perform indoor positioning and motion tracking and none of them can do both under low power, low cost and compacity constraints addressed by our platform. As Zyggie has the capability to estimate distances w.r.t other platforms in the environment and quaternions (which represents the attitude/orientation) users can test/enhance state of the art algorithms for positioning and motion tracking applications.
\end{abstract}

\section{INTRODUCTION}

Knowing the posture/location of a person in its environment becomes an important issue for applications providing services based on these criteria. Many solutions have been proposed going from satellite based techniques (i.e. GPS), video tracking or radio-electrical waves combining. Some of them use optical signals such as infrared, laser or video tracking approaches, some use radio signal information (Time Of Flight (TOF) or RSSI) and others, data provided by IMU. To choose the adequate component for the platform design and obtain the best trade-off for considered applications, a comparison in terms of accuracy, deployment facility, size, power consumption and price has to be done.

As both indoor positioning and motion tracking need low cost, portable and low power systems which can be deployed in minutes, radio and IMU are the best candidates. The main contribution of this paper is a platform named Zyggie that combines hardware components such as UWB chip for TOF distances measurement, $2.4 \mathrm{GHz}$ transceiver for RSSI sensing and an IMU for motion estimations.

Several Zyggie platforms are used to form a Wireless Sensor Network (WSN) which medium access has to be scheduled. To do so a custom Time Division Multiple Access Medium Access Control (TDMA MAC) has been developed to get the best trade-off between consumption and performance.

Another contribution is the data processing chain which is composed of some filtering and calibration phases to format data for positioning and motion tracking algorithms. Two GUIs have been developed for the this purpose. First a Matlab one for testing/validating algorithms, then an Android tablet one for displaying on a 3D avatar the results in real-time.

The rest of this paper is organized as follows. Section II summarizes state of the art platforms that propose either positioning and/or motion tracking capabilities. Section III introduces the hardware of our new developed platform named Zyggie. Data exchanges provided by Zyggie are then explained in Section IV using a custom TDMA MAC protocol. In Section V output data from Zyggie are used to evaluate positioning and motion tracking applications (section V-A \& V-B). Finally, conclusions are drawn and future works are commented in Section VI.

\section{RELATED WORK}

Table I lists most relevant solutions for either indoor positioning and/or motion tracking applications. These solutions are compared in terms of:

- Indoor Positioning and/or Motion Tracking capabilities,

- Accuracy of measurements,

- Deployment which represents the system setup facility and the flexibility toward the application,

- Size of wearable devices (if needed) and/or the associated system infrastructure,

- Power consumption,

- Price of the system.

Vicon system [1] is known as a reference in motion tracking applications due to its millimeter accuracy. Several infrared cameras are used to track retroreflective markers. This opticalpassive solution allows a long period of use but leads to significant computation resources and power needs.

Kinect [2] is a low cost general public markerless solution that embeds infrared and RGB cameras. Depth matrix coupled with image analysis enable the system to track in $3 \mathrm{D}$ space the attitude of a person. As Kinect has a unique camera viewpoint, it can lead in some cases to an information loss (i.e. arm hidden by the user).

Unlike video tracking solutions Xsens is only based on IMU. High quality hardware and complex processing algorithms needed for motion tracking applications lead to a good accuracy but high power consumption.

ShotTracker [3] is a basket system monitoring which allows the positioning of players and the ball in addition to scoring capability. This solution, based on UWB technology, is only 
dedicated to the previously mentioned application due to the fact that the system is fixed to the building.

Bluetooth Low Energy (BLE), ZigBee or other Radio Frequency (RF) technologies [4]-[9] can use received signal power to get distance information between network's nodes. Obtaining this RSSI is not consuming but is inaccurate (more or less $2.5 \mathrm{~m}$ ) due to its environment perturbations' sensitivity. This makes it not suitable for some of the applications.

The global view of Table I highlights the lack of a mobile, low cost, low power system with reasonable accuracy for both targeted applications. This lack is filled with Zyggie platform, detailed in Section III.

TABLE I

INDOOR POSITIONING AND MOTION TRACKING SOLUTIONS COMPARISON

\begin{tabular}{|c|c|c|c|c|c|c|c|}
\hline 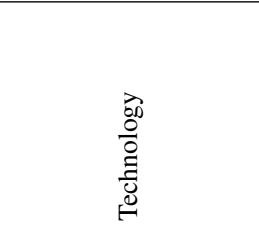 & 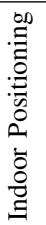 & 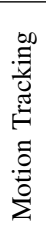 & 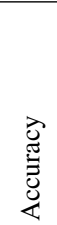 & 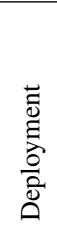 & $\stackrel{\tilde{N}}{\sim}$ & $\begin{array}{l}\overline{0} \\
\text { zे } \\
\text { aे }\end{array}$ & $\stackrel{\mathscr{E}}{\varrho}$ \\
\hline Video w/ marker [1] & $\overline{\overline{Y e s}}$ & 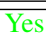 & 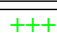 & $\overline{-\cdots}$ & 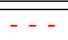 & $\begin{array}{ll}-- \\
\end{array}$ & $\overline{-\cdots}$ \\
\hline Video w/o marker [2] & Yes & Yes & - & + & ++++ & - & ++++ \\
\hline IMU [10] & No & Yes & ++ & - & + & -- & - \\
\hline UWB [3] & Yes & No & +++ & -- & + & + & - \\
\hline RSSI [4]-[8] & Yes & No & $\cdots$ & - & ++ & ++++ & +++ \\
\hline UWB / IMU / RSSI & $\overline{\mathrm{Yes}}$ & Yes & + & +++ & +++ & $\overline{+++}$ & +++ \\
\hline
\end{tabular}

\section{ThE Zyggie PlATFORM}

\section{A. UWB Technology}

UWB systems allow to emit low power and ultra-short duration pulses and provide applications with following advantages:

- High precision TOF ranging at centimeter level,

- Low electromagnetic pollution and reduced interference to other systems,

- Small size and low power consumption.

TOF computation is explained as following: a message is sent from the emitter to the receiver and after some processing time the receiver replies back. The TOF from the emitter to the receiver is the difference between the time that the emitter received the response from the receiver and the emission time divided by 2 , by taking care of the response time of the receiver. Thus, we can compute the distance $d$ depending on TOF with the following formula

$$
d=T O F \cdot c=\left(\frac{T_{r e}-T_{e m}-T_{r}}{2}\right) \cdot c,
$$

where $T_{e m}$ is the emission time, $T_{r e}$ the response reception time, $T_{r}$ the response delay of the receiver, and $c$ the celerity which is assumed to be the same as the light in air. Due to some drifts on the emitter and receiver frequencies/clocks, an error is present on the measurements. In order to minimize this error this scheme can be repeated twice.

There are only few vendors of UWB chipsets among which we can cite BeSpoon, DecaWave [11], Blinksight and Alereon. As DecaWave chips are the most open for experimentation (frequency band, datarate, transmitting power programming), they were chosen for our prototype (see Section III-B).

\section{B. Zyggie Node architecture}

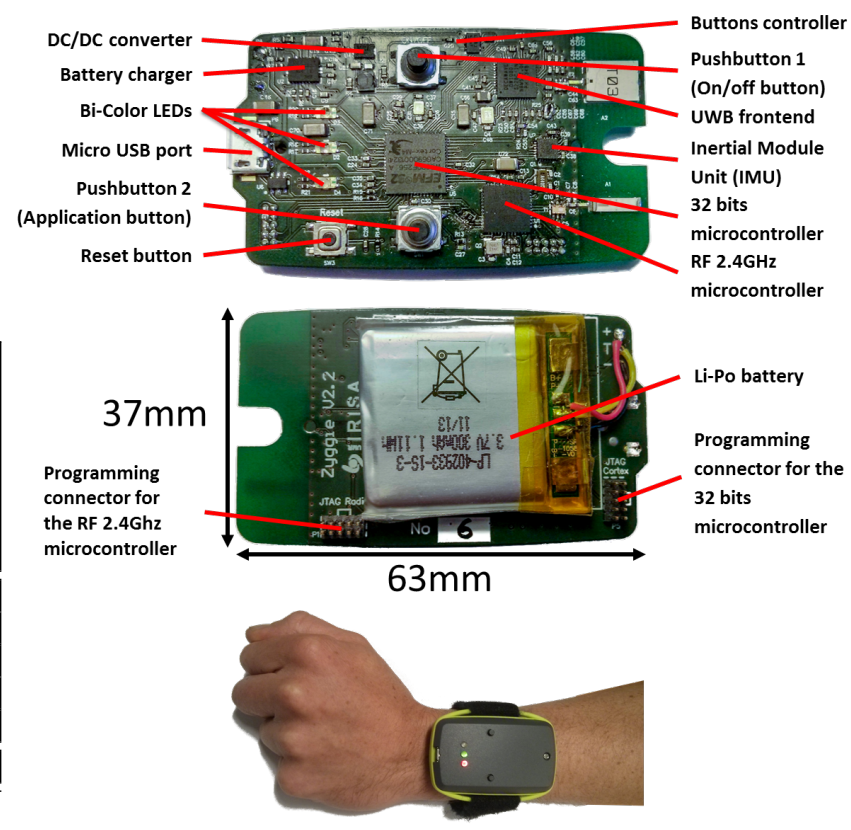

Fig. 1. Zyggie WSN Hardware illustration.

The Zyggie node is a Wireless Sensor Node with UWB radio capabilities that was developed in our lab. Its architecture is represented on Fig. 1 and composed of:

- A Silicon Labs EFM32 ARM CortexM4 micro-controller which is the Master Control Unit (MCU) of the node and also controls the UWB radio chip,

- An ATMEGA2564RFR2 which drives the $2.4 \mathrm{GHz}$ radio for data exchanges and network synchronization,

- A DecaWave DW1000 UWB radio chip [11] for TOF measurements,

- An InvenSense IMU for acceleration, angular speed and magnetic field captures.

Our node is ultra compact ( $37 \mathrm{~mm}$ by $63 \mathrm{~mm}$ ) and only weights $32 \mathrm{~g}$ (including battery) which makes it a real good candidate for all applications where weight and size are critical. Some experiments with UWB radio enabled at full speed were done leading to a battery life time of around 5 hours which is enough for sportsman or in building applications.

\section{Zyggie MAC PROTOCOL}

The network is composed of identical hardware Zyggie nodes. One of them is the central node (coordinator) connected to a computer/tablet, the others are on-body nodes and, if necessary, off-body fixed nodes for positioning. Coordinator aggregates data from all Zyggie nodes $\left(Z_{i}\right.$ with $i \in[1, N]$ and $N$ the number of nodes) to process and plot them on GUI 


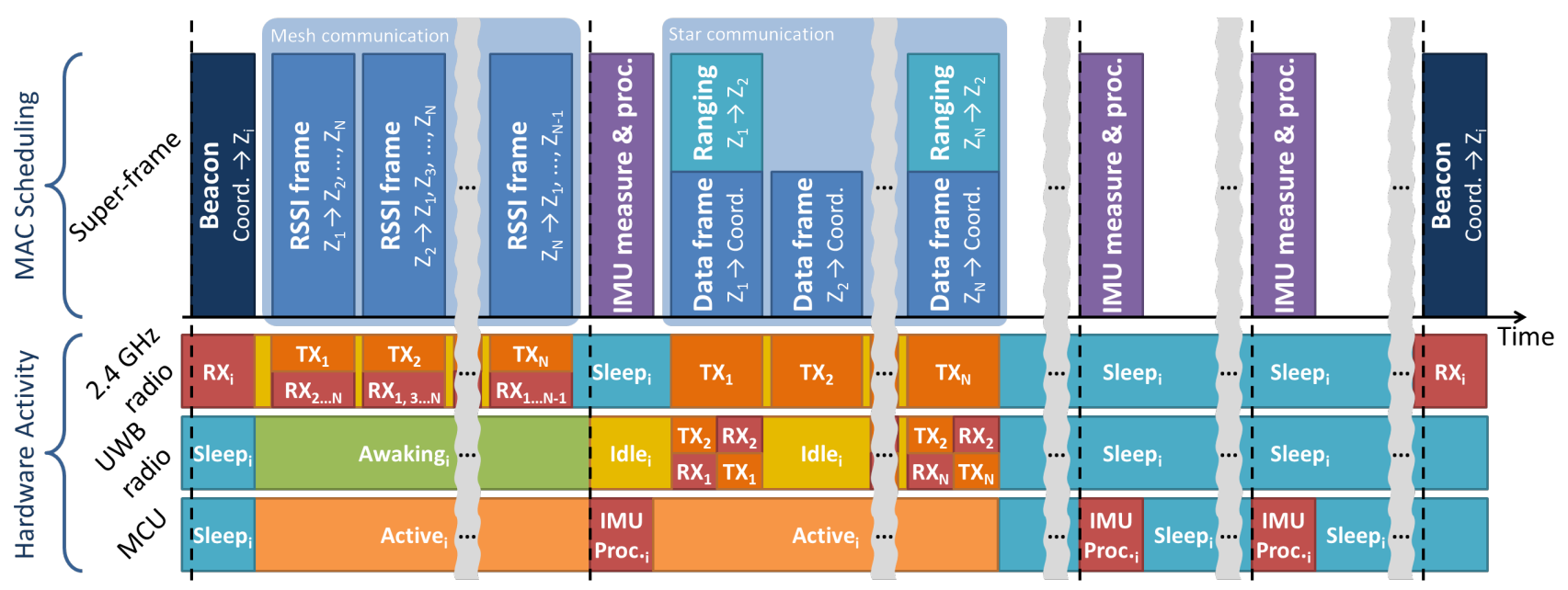

Fig. 2. Custom TDMA MAC

presented in Section V-B. A custom TDMA MAC protocol allocates time slots to each node to allow it sending its sensed data. All nodes have to sense movement data like IMU information or system absolute orientation, RF messages energy called RSSI and distance estimation with TOF measurements. Our MAC is based on the IEEE 802.15.4 communication protocol with a beacon-enable network. The coordinator broadcasts periodically a beacon frame to synchronize nodes and share network information. As shown on Fig. 2, a full mesh communication is started to get RSSI information. In its RSSI time slot, each node sends a short broadcast frame (orange TX slots) that others will listen (red RX slots) to get a complete RSSI matrix. As the IMU measurement step starts (purple time slot), the MCU gets data (red IMU Processing slots) and optionally performs an Extended Kalman Filtering (EKF) to produce a quaternion. Each node in its time slot (Data frame time slot) sends these data via the $2.4 \mathrm{GHz}$ radio transceiver to the coordinator. During this star communication phase, nodes also process TOF ranging measurements. First, the UWB chip has to be awaken (green Awaking slot), then it can wait to be used (yellow Idle slots) and finally it does its ranging (orange \& Red TX/RX slots) before returning to sleep. In the example of Fig. 2 the node 2 is selected to do ranging toward others, therefore a distances' vector can be sent to the coordinator at its next Data frame. A sequence of selected ranging nodes has to be programmed to get a full distances matrix after $N$ beacons. Previously defined phases are scheduled to get the best trade-off between consumption and performance, that is the reason why transceivers spend a minimum time into receiving mode. On the other hand, an adaptive data-rate is implemented depending on the number of nodes, to deal with the processing and communication time.

\section{PROOF OF CONCEPT}

As previously said, our platform can output data that can be used by users to test algorithms in the context of positioning and motion tracking. To be user friendly, we have also developed a GUI modeling an avatar in 3D space to visualize the results. These two aspects are now going to be explained in more details.

\section{A. Algorithms performance evaluation}

Algorithms take part of the processing chain as shown in Fig. 3. On the chain at the top of the figure, UWB distances allow to deal with positioning application. On the chain at the bottom of the figure, computed information coming from IMU (i.e. quaternions) and UWB distances allow to deal with motion tracking application. For both applications, it exists state of the art algorithms (coloured boxes at the end of the chains) but users can also develop and/or optimize these algorithms. Blocks involved in these chains are now going to be presented.

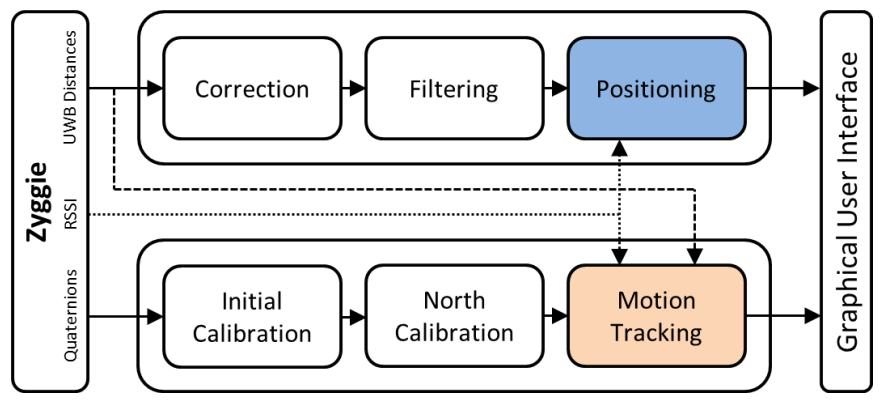

Fig. 3. Zyggie data processing chain

1) Positioning: As we are able to obtain a measured distance between two nodes the next step is to compute several distances between a mobile node and some fixed nodes named anchors. The process chain starts with some corrections depending on antenna delays and hardware design. Then as TOF measurements can be noisy, some filtering is done to remove aberrant values. Finally, to obtain the coordinates of the mobile a localization algorithm is used (blue Positioning box in the chain) among we can cite centroid, trilateration, multilateration, Gauss-Newton [12]-[14] and so on. Users can 
also test their algorithms with data provided by Zyggie (i.e. UWB distances and/or RSSI).

For instance, we experimented 2D positioning of a walking man using Zyggie with a custom low computation noise-aware algorithm based on trilateration. For this experimentation, data were acquired in a room of nearly $243 \mathrm{~m}^{2}$ (26.4 m length by $9.2 \mathrm{~m}$ width). The ideal trajectory was marked on the floor and one step by second (around $3 \mathrm{~km} / \mathrm{h}$ ) was done following this trajectory. In order to test our algorithm a six anchors configuration is used. Fig. 4 represents the computed trajectory by our algorithm in red and the ideal one in green.

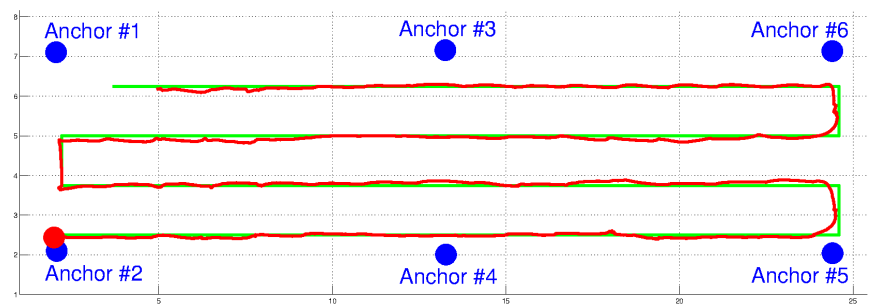

Fig. 4. Results for indoor walking context

2) Motion tracking: First, we implemented the proprietary InvenSense $\mathrm{EKF}$ on the 32bits $\mathrm{MCU}$ in the Zyggie platform to send less information via RF. This data fusion algorithm converts raw IMU data (i.e. acceleration, rotation and magnitude) into quaternions to represent absolute system orientation on 3 axes. Then, user needs to do 2 specific postures for Initial and North calibrations (the first two boxes in the bottom chain of Fig. 3). Classical "T" and "I" postures were chosen to measure arm movements and calibrate user's posture. When arms are raised to form a "T", all quaternions are saved to achieve the computation of

$$
q_{\text {init }_{\text {joint }}}=q_{\text {raw }_{\text {joint }}} \cdot q_{0_{\text {joint }}}^{-1},
$$

where joint represents the joint name associated to a node,

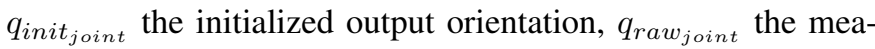
sured orientation and $q_{0_{\text {joint }}}$ the initial quaternion equals to $q_{\text {raw }}^{-1}$ only during the first calibration posture. After the second posture done, we select one arm node (i.e. left forearm) to resolve the System (3) and find the $q_{\text {north }}$ quaternion.

$$
\left\{\begin{array}{l}
q_{T}=q_{\text {north }}^{-1} \cdot q_{T} \cdot q_{\text {north }} \\
q_{I}=q_{\text {north }}^{-1} \cdot q_{\text {init }_{L F}} \cdot q_{\text {north }}
\end{array},\right.
$$

where $q_{T}$ is the quaternion to form the "T" pose, $q_{I}$ the wanted quaternion to form the "I" pose and $q_{i n i t_{L F}}$ the measured and initialized quaternion for the "I" pose.

This system is in the form of the Wahba's problem therefore a solution can be found with Singular Value Decomposition (SVD) as described in [15]. Solutions can be found with the other arm nodes to get a single quaternion, therefore reducing the measuring noise due to both the sensor and user approximate posture.

Finally, depending on the targeted application, user's movement or posture can be processed easily with data (i.e. quater- nions and/or RSSI and/or UWB distances) provided by Zyggie (orange Motion Tracking box in the chain).

\section{B. Graphical User Interface}

These algorithms were implemented into two GUIs for different needs. First of all, Matlab was used to make acquisitions and work on raw data. It allows us to display in real-time raw data like RSSI and UWB distances table and accelerometer, gyroscope and magnetometer graphics. Other features are available for posture and movement recognition with Principal Component Analysis (PCA) classification algorithm [16]. Targeted applications are in medicine for functional rehabilitation of a patient or in Human-Machine interaction with gesture.

Then, an Android tablet application was developed for demonstrations to show the system ability in a mobile context. Features like IEEE 802.15.4 channel scanning at $2.4 \mathrm{GHz}$ and $3 \mathrm{D}$ avatar tracking were added. Positioning algorithms were also included to update avatar position in a 3D environment as displayed on Fig. 5.

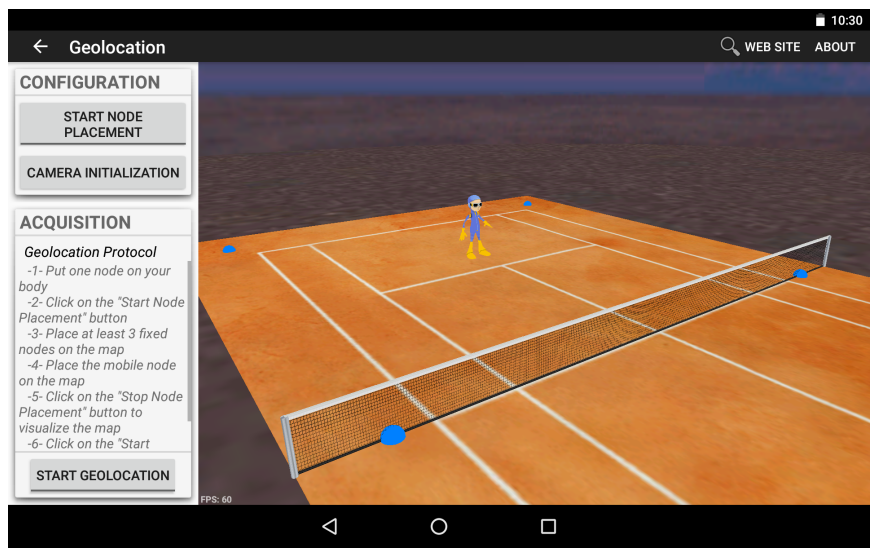

Fig. 5. Android application: blue dots are the anchors for positioning and the tennis player is also equipped with nodes for motion tracking.

\section{CONCLUSION}

We have proposed in this paper a new low power, low cost hardware/software platform named Zyggie for indoor positioning and motion tracking applications. Zyggie combines UWB technology for accurate indoor positioning and IMU for motion tracking; RSSI metrics can also be inputted for both applications. Our prototype has been validated in real use cases (indoor human tracking). An Android application has also been developed for real-time visualization on a tablet. Finally Zyggie offers the opportunity for users to test their own developed algorithms in the Matlab environment.

\section{ACKNOWLEDGMENTS}

This work has been conducted in the context of the Cominlabs Labex BoWI project [17] funded by the "Investissement d'avenir" program. 


\section{REFERENCES}

[1] "Vicon system." [Online]. Available: https://www.vicon.com/

[2] Z. Zhang, "Microsoft kinect sensor and its effect," IEEE MultiMedia, vol. 19, no. 2, pp. 4-10, Feb 2012

[3] B. C. Ianni, D. D. Ross, C. A. Kahler, and D. A. Danknick, "Basketball shot-tracking system," Sep. 8 2015, uS Patent 9,129,153.

[4] N. L. Dortz, F. Gain, and P. Zetterberg, "Wifi fingerprint indoor positioning system using probability distribution comparison," in the IEEE International Conference on Acoustics, Speech and Signal Processing (ICASSP), March 2012, pp. 2301-2304.

[5] M. Shchekotov, "Indoor localization methods based on wi-fi lateration and signal strength data collection," in the 17th Conference of Open Innovations Association (FRUCT), April 2015, pp. 186-191.

[6] Y. Wang, Q. Yang, G. Zhang, and P. Zhang, "Indoor positioning system using euclidean distance correction algorithm with bluetooth low energy beacon," in the International Conference on Internet of Things and Applications (IOTA), Jan 2016, pp. 243-247.

[7] S. Fang, C. Wang, T. Huang, C. Yang, and Y. Chen, "An enhanced zigbee indoor positioning system with an ensemble approach," IEEE Communications Letters, vol. 16, no. 4, pp. 564-567, April 2012.

[8] L. Luoh, "Zigbee-based intelligent indoor positioning system soft computing," Soft Computing, vol. 18, no. 3, pp. 443-456, 2014.

[9] A. Aulery, C. Roland, J.-P. Diguet, Z. Zheng, O. Sentieys, and P. Scalart, "Radio signature based posture recognition using wbsn," in the 14th
International Conference on Information Processing in Sensor Networks (IPSN), 2015, pp. 322-323.

[10] D. Roetenberg, H. Luinge, and P. Slycke, "Xsens mvn: full 6dof human motion tracking using miniature inertial sensors," Xsens Motion Technologies BV, Tech. Rep, 2009.

[11] "Decawave uwb chipset." [Online]. Available: http://www.decawave. com/products/dw1000

[12] J. Blumenthal, R. Grossmann, F. Golatowski, and D. Timmermann, "Weighted centroid localization in zigbee-based sensor networks," in IEEE International Symposium on Intelligent Signal Processing (WISP), Oct 2007, pp. 1-6.

[13] T. He, C. Huang, B. M. Blum, J. A. Stankovic, and T. Abdelzaher, "Range-free localization schemes for large scale sensor networks," in the 9th International Conference on Mobile Computing and Networking (MobiCom). ACM, 2003, pp. 81-95.

[14] J. Nocedal and S. Wright, Numerical Optimization: 10.3 Algorithms for Nonlinear Least-Squares Problems. Springer New York, 2006.

[15] D. M. Bates, M. J. Lindstrom, G. Wahba, and B. S. Yandell, "Gcvpack routines for generalized cross validation," Communications in Statistics - Simulation and Computation, vol. 16, no. 1, pp. 263-297, 1987.

[16] A. Aulery, J. P. Diguet, C. Roland, and O. Sentieys, "Low-complexity energy proportional posture/gesture recognition based on wbsn," in the 12th International Conference on Wearable and Implantable Body Sensor Networks (BSN), June 2015, pp. 1-6.

[17] "Cominlabs labex bowi project: Body world interaction." [Online]. Available: http://www.bowi.cominlabs.ueb.eu 